\title{
"Silence lourd et blanc comme une colère qui monte ": donner corps au silence dans La Chambre des enfants de Louis-René des Forêts
}

Le silence tient une grande place dans l'œuvre de Louis-René des Forêts. Pascal Quignard intitule son livre portant sur l'œuvre de Louis-René des Forêts Le Vou de silence (1985). Il y développe l'idée que Louis-René des Forêts écrit pour faire entendre le silence, c'est-à-dire pour dénoncer les insuffisances du langage, lequel est toujours associé à une compromission de l'individu. Seul le silence serait capable de rendre compte de la singularité des choses, singularité perdue dès lors qu'on nomme l'objet par un nom qui ne décrit pas que lui - un nom qui n'est pas un nom "propre ", un nom toujours plus ou moins impropre, «commun » dans son sens péjoratif de «banal », « sans intérêt ».

Cependant, le livre de Marc Comina, Louis-René Des Forêts, l'impossible silence (1998) renverse la perspective, considérant que si de nombreux critiques - Pascal Quignard, Maurice Nadeau, Bernard Pingaud - avaient lu chez cet auteur une dénonciation des manques du langage, c'est à cause de la fascination que leur inspirait le personnage de l'écrivain, reculé, dont les rares périodes de création laissaient place à de longues plages de silence. Marc Comina montre d'abord qu'il n'en est rien, que Louis-René n'a cessé d'écrire, et que de plus, le silence apparait dans son œuvre comme « un refuge » mais qui fait courir à celui qui s'y abrite le risque de la solitude :

S'il est indéniable que le silence peut servir de refuge à celui qui a trop parlé, il n'en est pas moins vrai qu'il s'agit d'un abri provisoire, dans la mesure où il possède le défaut majeur de couper l'homme du lien vital avec ses semblables. Ainsi, comme on peut souhaiter quitter la parole pour retourner au silence, il vient un moment où le besoin de parler avec autrui l'emporte sur toute autre considération (Comina, 1998:12).

Marion Coste - ATER à l'IUT Robert-Schuman, Illkirch. Adresse pour correspondance : IUT Robert-Schumann, 72 route du Rhin, 67400 Illkirch ; e-mail : marioncoste88@gmail.com 
Cette phrase s'adapte parfaitement à La Chambre des enfants. Dans ce récit, un adulte épie derrière une porte des enfants en train de jouer à faire parler l'un d'eux, Georges, décidé à rester mutique. Ils proposent d'abord des jeux de rôle complexes, faisant mine de parler à la place de Georges, puis décident de se taire eux-mêmes pour que le silence devienne insupportable à Georges. C'est alors l'adulte qui ne le supporte plus et interrompt la scène, avant de se souvenir qu'il s'appelle Georges et d’être réveillé par l'un des enfants. Dans ce récit, par le biais des métaphores, le silence prend corps, jusqu'à devenir une présence intolérable pour le narrateur, un ennemi contre lequel il faut absolument se prémunir par la parole.

Je me demanderai comment Louis-René des Forêts fait du silence un personnage avec lequel le narrateur doit négocier.

D'abord, je verrai que le silence est un allié qui confère à l'enfant mutique une qualité de présence qui manque aux autres, puis je verrai que lorsque tous les enfants se taisent, le silence prend corps, devient un « agresseur » contre lequel il faut se défendre.

\section{Le silence : un allié puissant de l'enfant}

Georges est mutique. Il est seul contre une bande d'enfants qui souhaite le faire parler, mais sort victorieux de cet affrontement. Il semble donc que le silence soit un allié nécessaire et puissant pour affronter la foule des autres enfants. On retrouve là un schéma classique dans les récits de Louis-René des Forêt : qu'on pense à "Une mémoire démentielle », par exemple. L'opposition entre le groupe d'enfants et Georges est présentée comme une lutte :

- On dirait pourtant qu'il est venu ici pour soutenir une épreuve et nous pour la lui faire subir.

- Pas du tout. Est-ce qu'il n'a pas l'air à son aise ?

- Bien plus que nous en effet. On l'a offensé, mais c'est nous qui le sommes par son silence (Des Forêts, 1960 : 67-68).

Le pronom indéfini " on ", repris par «nous ", fait du groupe d'enfants une identité plurielle, difficile à cerner mais soudée contre Georges, qui est toujours nommé par la troisième personne du singulier : « il », « lui », « l' ». Le verbe "offensé » appartient à un langage châtié assez étonnant dans la bouche des enfants : il évoque l'univers de la chevalerie, ce qui peut suggérer que se joue ici un combat symbolique essentiel, entre le bien et le mal, comme celui que menaient les chevaliers. L'expression " soutenir une épreuve » évoque quant à elle les martyrs chrétiens, ce qui tend aussi à donner à ce combat d'enfants des allures de lutte morale, entre le bien et le mal. Cependant, les enfants reconnaissent ici la supériorité de Georges. Alors même qu'il a été offensé (" on l'a offensé », au présent de l'indicatif : l'action a eu lieu, sans 
aucun doute), l'offense s'est retournée contre les offenseurs, grâce au silence. Dans "c'est nous qui le sommes par son silence ", la forme passive ainsi que le complément d'agent «par son silence » montrent que ce n'est pas Georges qui s'est défendu, mais qu'il a été défendu "par son silence ", comme il aurait pu l'être par son allié. En effet, Georges a fait vœu de silence. Un enfant dit : «Et il a eu bien raison de venir, car en refusant il aurait rompu son vœu. " (Des Forêts, 1960 : 69). Le mutique est donc un personnage noble, prestigieux, qui a fait vœu de silence, comme un moine ou un ascète : l'intertexte chrétien, très présent chez Louis-René des Forêts, fait du mutique un personnage supérieur aux autres enfants. Cette supériorité est confirmée par le narrateur :

Or, de tous les enfants enfermés dans la chambre, c'est encore ce Georges dont la présence lui semble la moins contestable, car il bénéficie du prestige de son mutisme quand les autres, en s'empruntant mutuellement leurs voix, paraissent se confondre en une seule et même personne, toujours versatile, toujours indéterminée (Des Forêts, 1960 : 68).

L'image des enfants « s'empruntant mutuellement leurs voix » évoque une hydre, qui a plusieurs têtes (donc, potentiellement, plusieurs voix) mais un seul corps. Elle est l'un des ennemis fréquents des dieux (Zeus) ou des héros (Hercule), et symbolise, elle aussi, les forces du mal. Cette métaphore insiste sur l'infériorité du groupe d'enfants, dont la présence est même mise en doute. Elle vient du fait que le narrateur entend seulement la voix de Paul, son neveu, imitant les voix des différents enfants qu'il nomme. Ainsi, quand Paul interpelle une certaine " Jeannette », le narrateur pense :

Jeannette ? Mais il ne reconnaît pas sa voix et quant à celle de Paul, elle répond aux propos tenus par lui avec la voix d'un autre. Paul jouerait-il donc à tenir tour à tour le rôle de chacun des interlocuteurs supposés, mais alors dans quel but tient-il celui des autres avec sa propre voix et le sien avec celle d'un autre? (Des Forêts, $1960: 68$ ).

Les enfants sont déréalisés : on ne sait pas combien ils sont, ni s'ils existent réellement ou sont seulement des rôles. À ces enfants bavards s'associe la métaphore du théâtre, elle aussi structurante dans les récits de Louis-René des Forêts. Ainsi Paul, comme un acteur, joue peut-être à " tenir tour à tour le rôle de chacun des interlocuteurs supposés ». Ils préparent même une sorte de décor et de scène :

Si on s'interpelle, si on marche, c'est avec la plus grande discrétion, comme le feraient, entre deux tableaux, des machinistes derrière un rideau de fortune. [...] Amplifiée par la majesté du ton, la voix de Paul parait tomber de très haut, comme d'une montagne ou d'un balcon, et il est bien possible que, pour mieux dominer son auditoire, Paul ait inventé de se jucher pour la circonstance sur un échafaudage de meubles hâtivement dressés, sur ses instructions, par les autres enfants (Des Forêts, 1960 : 71). 
Les « tableaux », les « machinistes » et le « rideau » évoquent l'univers de l'opéra, d'ailleurs largement développé dans "Les Grands moments de la vie d'un chanteur ", récit qui précède, dans notre recueil, celui qui nous intéresse. Paul se met en scène, sur une sorte d'estrade de fortune. Leurs paroles sont toujours soumises au doute du narrateur : on ne sait pas qui parle, et même les enfants ne semblent pas très sûrs de ce qu'ils disent. Ainsi, Paul expose une théorie selon laquelle les enfants apprécient de se voir infliger une punition, ce qui mène à la dissolution même du langage :

- Tu n'aimes pas les punitions, mais c'est un fait que tu aimes qu'on te les inflige, méritées ou non!

- C'est faux!

- Mettons que Paul dise vrai et voyons où il veut en venir.

- À ceci... Mais vous m’avez égaré ! Je t’en prie, Georges, viens à mon secours, toi qui as suivi mon raisonnement sans m'interrompre!

- Comme si Georges allait se laisser prendre à une ruse aussi grossière! (Des Forêts, $1960: 72-73)$.

D’abord, la proposition de Paul est réfutée : «C’est faux !». Ensuite, elle est acceptée en tant qu'hypothèse : "Mettons que Paul dise vrai et voyons où il veut en venir. " Le subjonctif déréalise l'énoncé. Paul avoue ensuite qu'il a perdu le fil de son raisonnement, et appelle à son aide le mutique, seul capable de suivre jusqu'au bout une pensée. Le langage, à travers Paul, admet son infériorité au silence. Mais cet appel à l'aide est lui-même remis en question, puisqu'un enfant l'analyse comme une ruse pour faire parler Georges. Le langage n'est plus capable de dire le monde : chaque parole est douteuse, mensongère, manipulée. Seul le silence de Georges est capable de sincérité.

Jusqu'ici, le récit semble bien associer au langage, aux enfants qui parlent, l'univers du théâtre, du faux, du mensonge ; au contraire, l'enfant mutique est considéré comme un chevalier, un combattant, un moine ou un martyr. Il est du côté de la vérité et du refus de la compromission. L’analyse de Pascal Quignard, dans Le Voeu de silence, semble très éclairante :

Schéhérazade dit qu'il faut garder scrupuleusement les secrets et les vœux. Que si nous n'avons pas pu contenir en notre sein une confidence, aucun de ceux auxquels nous la confierons ne saurait la contenir. « Tout être, ajoute-t-elle, qui ne desserre pas les lèvres est un être qui est hors de danger. Le silence est la beauté qui orne les plus belles choses du monde. Toute parole est semblable à la pluie d’orage qui gâte tout. » (Quignard, 1985 : 54).

Le silence, dans ce récit, est un refuge pour ceux qui refusent la compromission du langage. Le mutique fascine tout le monde : les enfants ne cessent de commenter le comportement de ce Georges. Ils interprètent ses expressions : 
- Et moi, son visage me dit qu'il est offensé.

- Quelle imagination! Je ne lui trouve aucune expression, sinon de l'ironie. Il s'amuse à nos dépens, et nous sommes bien sots de lui en donner l'occasion ! (Des Forêts, $1960: 69)$.

- [...] Bravo, Paul! Tu as réussi à faire sourire Georges !

- Georges sourit de ton ignorance! (Des Forêts, 1960 : 72).

Ils reconnaissent « épiloguer sur sa présence » (Des Forêts, 1960 : 69) et ne cessent de faire des hypothèses sur ce qu'il pense : "Je me demande si Georges admet qu'on puisse ouvrir la bouche et rester muet. / Son silence prouve qu'il ne l'admet pas. » (Des Forêts, 1960 : 81).

Cependant, il y a deux mutiques dans ce récit : Georges, mais aussi le narrateur qui épie derrière la porte. Si l'un est prestigieux, l'autre, au contraire, ressent de la honte. Le récit commence par l'évocation de ce sentiment de honte, mystérieux et impérieux :

Rien ne saurait donner une idée de la stupéfaction, de la honte qu'il éprouve à se tenir planté indiscrètement derrière la porte entrouverte de la chambre des enfants. [...] il sétonne surtout de ce malaise qu'il juge hors de proportion avec sa cause, car enfin, s'il est vrai que jamais avant ce jour il ne s’était hasardé jusqu’à la porte de la chambre des enfants ni probablement aucun des enfants jusqu'à la sienne, quel scrupule moral, quelle convention domestique le lui auraient interdit? Et maintenant qu'il se trouve comme fortuitement derrière cette porte, c'est pour s'étonner que ce trouble indéfinissable qui ne relève en rien du sentiment de transgresser une loi, de violer un secret, de se livrer à une activité équivoque le maintienne impérieusement à son poste au lieu de l'en arracher ; [...] (Des Forêts, 1960 : 65).

Ces lignes ouvrent le récit. La sensation de culpabilité est le déclencheur de l'action : c'est parce qu'il se sent coupable sans comprendre pourquoi qu'il continue à écouter. Au mutisme triomphant de Georges répond celui, coupable, du narrateur. Le silence est l'allié de Georges, mais il nuit au narrateur adulte. Ces deux facettes du silence se rejoignent, d'autant plus que Georges et le narrateur sont en fait une seule et même personne : le narrateur rêve, et Georges est une projection de lui enfant. On rejoint alors les analyses de Marc Comina, pour qui le silence protège et isole à la fois, conduisant donc nécessairement à la parole. De fait, le silence va se faire menaçant.

\section{Le silence ennemi}

La situation se retourne lorsque les enfants renoncent à la parole, et utilisent contre Georges le silence :

- Conviens de ton échec, avoue que tu avais espéré faire parler Georges en le soumettant comme nous à l'épreuve de tes ennuyeux sophismes, mais Georges l'a subie 
sans mot dire, s'il a paru se prêter un instant à ton jeu au cours de cette pittoresque scène de collège où tu lui avais attribué le rôle du professeur qu'il n'a tenu que par des grimaces! Conviens de ton erreur, Paul ! Condamnons maintenant Georges à subir notre mutisme et tu verras qu'excédé à son tour il cherchera à le rompre et n'y parviendra qu'en renonçant au sien!

- Voilà Georges averti ! Comment perdrait-il à un jeu dont tu lui étales imprudemment les cartes sous les yeux?

- Alors parce que je fais bon marché de tes piètres roueries, des chausse-trapes vraiment trop voyants de tes laborieux discours, je perdrais la partie ? Quelle naïveté de croire que mes paroles aient le pouvoir de durcir Georges dans sa position, quand les tiennes n'ont pas eu celui de l'en chasser ! (Des Forêts, 1960 : 85).

L'interlocuteur de Paul le somme de renoncer à la parole, associée au mensonge et à la manipulation sournoise ( "piètres roueries ", " chausse-trapes »). La parole ne peut pas mettre fin au mutisme. L'enfant déclare : "Chacune de vos paroles l'enfonce un peu plus dans son mutisme. » (Des Forêts, 1960 : 87). Il nie le pouvoir de la parole dans la dernière phrase de l'extrait. La stratégie du silence est explicitée un peu plus loin :

- Comprenez donc que si Georges a refusé de communiquer avec nous qui lui tenions un langage dont nous nous étions fait illusoirement une arme contre son mutisme, c'est qu'il lui était sans doute facile d'opposer son mutisme à nos bavardages, mais qui pourrait longtemps supporter en silence la société de taciturnes, sinon dans une communauté où le silence est de règle ? Or cette chambre n'a jamais été que par fiction le lieu où s'exerce le règlement ! Pourquoi parler encore de pièges, de contraintes, de résistance, de défaites? Je dis que c'est en connaissance de cause et comme poussé par la nécessité que Georges sortira de son mutisme pour nous arracher au nôtre, je dis qu'on ne saurait l'accuser de parjure puisqu'il ne renoncerait à son vœu qu'en acceptant de se livrer avec nous à un échange de paroles frauduleuses dont nous serions tous les dupes. Sa première parole, nous ne la lui extorquerons pas cette fois à la faveur de quelque artificieuse démonstration, elle naîtra spontanément de son mutisme comme un appel jeté à notre mutisme, aussi déchirant qu'une prière, aussi impérieux qu’un cri du cœur! (Des Forêts, 1960 : 86).

C'est au nom de la constitution d'une " communauté » que Georges devrait être amené à rompre son vœu de silence : la thèse de Marc Comina nous guide dans cette interprétation. En effet, les marques de la première personne du pluriel confirment l'idée que la parole vient au secours d'un « nous » qui ne peut perdurer dans le silence. " Georges sortira de son mutisme pour nous arracher au nôtre ", " il ne renoncerait à son vœu qu'en acceptant de se livrer avec nous à un échange de paroles frauduleuses dont nous serions tous les dupes " : j'ai souligné les mots qui portent l'idée d'une communauté pour en faire ressortir l'omniprésence. Le silence commence à prendre corps dans cet extrait, puisqu'il semble une sorte de monstre qui tient sous son em- 
prise le groupe d'enfants, et dont Georges (dont le nom d'archange n'a peut-être rien d'anodin) doit triompher, afin "d'arracher » au dragon ses victimes. Il est aussi une entité à laquelle on peut adresser " une prière » dans la dernière phrase. Avec une ironie indubitable, qui pourtant ne cache pas tout à fait la dimension sérieuse et symbolique du jeu, un enfant récalcitrant, qui ne souhaite pas se soumettre au jeu du silence proposé, déclare : "Oh saint Georges le Taciturne ! Secourez-nous dans notre détresse ! Nous avions la parole, on nous la retire pour en avoir abusé et c'est à vous de nous la rendre, ainsi soit-il! » (Des Forêts, 1960 : 88).

La parodie du discours liturgique est évidente. Elle confirme l'assimilation de Georges à Saint-Georges, ce qui fait du silence le dragon qu'il doit terrasser. Un peu plus loin, un enfant déclare : "Georges parlera, Georges nous sauvera tous! » (Des Forêts, 1960 : 89). Il semblerait donc que le groupe d'enfants devient silencieux pour que Georges prenne conscience du danger de solitude que représente le silence. Le point de vue narratologique change alors, et semble nous donner accès à l'intériorité de Georges. Ou plutôt, le narrateur adulte et Georges fusionnent. Dans le passage suivant, j'ai mis en italiques les propos qui décrivent l'attitude du narrateur adulte, et ceux qui renvoient plutôt à Georges.

De même qu'il n'aura pu quitter son poste aussi longtemps que les voix des enfants se seront fait entendre derrière la porte, de même il éprouve maintenant jusqu'au vertige la fascination de leur mutisme et communie avec eux dans l'espoir de leur délivrance qui sera ainsi comme la sienne. Chacun d'eux a parlé pour lui, mais c'est comme si lui-même eût parlé pour chacun d'eux avec une véhémence qu'il juge disproportionnée à l'insignifiance de ce colloque extravagant qui n'était sans doute qu'une fine parodie de la verbosité prétentieuse des maitres. Les enfants ayant cessé de parler, c'est comme s'il eût renoncé lui-même à parler, mais alors sa présence derrière la porte est sans objet et ne lui semble motivée que par l'imminence d'un dénouement (Des Forêts, $1960: 89$ ).

Cette fusion du narrateur adulte et de Georges fait que le narrateur adulte se sent la responsabilité de rompre le silence :

Ce sera donc à lui seul de l'affronter [le silence] et de le rompre pour assurer leur salut en même temps que le sien, et non pas à ce Georges qui, en partageant leur sort, a perdu le bénéfice de son mutisme qu'il ne devait quà leurs paroles (Des Forêts, $1960: 89$ ).

Les verbes « affronter » et " assurer leur salut » installent un léger registre épique, qui renvoie de nouveau au combat de Saint Georges avec le dragon. La personnification du silence s'affirme tant et plus :

Le silence se dresse alors devant lui comme un formidable agresseur que chaque instant fortifie. Silence qui devient bientôt si volumineux qu'il semble près de faire éclater l'espace trop réduit de la chambre où on le tient confiné, silence lourd et blanc comme une 
colère qui monte, silence qui, cessant d'être celui d'enfants sages ou trop rusés, est désormais le sien, rien que le sien ! (Des Forêts, 1960 : 89-90).

L'image du dragon est suggérée par le verbe "se dresse ", par «formidable ", à prendre ici dans son sens étymologique de "terrible ", " effrayant ». L'anaphore, dans la deuxième phrase, du mot "silence", sans déterminant, lui donne quelque chose de mythique, le transformant presque en nom propre. Le narrateur insiste sur la présence physique du silence, qui tient trop de place (« si volumineux qu'il semble près de faire éclater l'espace trop réduit de la chambre »), un poids ( «lourd ») et une couleur ( "blanc»). Le silence, qui semblait être un jeu ou une bravade d'enfant, révèle ici sa nature maléfique. L'image du dragon, qui est l'incarnation du diable et l'ennemi traditionnel des archanges, montre que le silence est une tentation dangereuse. Le narrateur s'oppose à ce monstre, en faisant irruption dans la chambre : «Êtes-vous là, les enfants ? s"écrie-t-il en se jetant sur la porte dans un mouvement excédé. Êtesvous encore là ?» (Des Forêts, 1960 : 90).

Cette question répétée de la présence des enfants est révélatrice du fait que, comme le remarque Marc Comina, la véritable hantise de l'œuvre de Louis-René des Forêts est la solitude, bien plus que le silence. Le silence est un dragon qui isole l'homme de ses semblables, alors même qu'il semble lui conférer la toute-puissance : la dimension diabolique, méphistophélique, de ce silence devient lisible. Cette mise au jour des dangers du silence était peut-être le dessein de ce récit, puisque, après ce paragraphe, le dénouement est extrêmement rapide, voire légèrement décevant : le narrateur se réveille, tout cela n'était qu'un rêve :

C’est alors qu'il revint à lui pour se souvenir avec allégresse que son nom était Georges. Ouvrant les yeux, il vit le jeune Paul faire irruption dans sa chambre. Il le vit se pencher à son oreille afin de l'informer en souriant que quelqu'un, en bas, demandait à lui parler.

"Me parler, dis-tu, mon petit ? Me parler à moi ?"

Et, comme il se levait en hâte, il vit encore l'enfant lui sourire, mais ce sourire était lointain, indéchiffrable (Des Forêts, 1960 : 89-90).

Le réveil est un réveil heureux, comme l'indique le complément circonstanciel « avec allégresse. » Il se fait dans une mise en abyme de la parole : Paul parle au narrateur pour l'informer que quelqu'un veut lui parler. Cette requête semble flatter le narrateur, ou en tout cas l'aider à s'affirmer, grâce à la redondance des marques de première personne du singulier « Me parler à moi » (je souligne). La parole, après la révélation de la monstruosité du silence, devient le moyen d'un lien à autrui et de l'affirmation de soi.

Cependant, le récit se conclut sur une image du silence, avec cet enfant au sourire " lointain », « indéchiffrable ». Il s'agit bien, comme le dit Marc Comina, d'une dialectique entre parole et silence, l'un entraînant toujours l'autre : le silence vient purger les bavards d'un excès de parole, mais leur fait courir le risque de la solitude, poussant alors chacun à le briser. 


\section{Conclusion}

Ce récit montre donc un double risque : celui de la parole, fallacieuse, mensongère, stérile, et celui du silence, magnifique, agressif, solitaire. Le silence est associé à une créature tentatrice, capable de conférer la toute-puissance au mutique, mais lui faisant risquer la solitude. Dans ce récit, il prend corps et révèle son caractère méphistophélique, poussant le narrateur à le combattre pour sauver in extremis la communauté, c'est-à-dire son lien avec ses semblables.

Une autre interrogation, liée à celle du silence et de la parole, traverse le récit : c'est celle de la stabilité des identités. Si je n'ai pas explicitement traité cet aspect, les quelques extraits de texte ci-dessus permettent de comprendre que toutes les identités sont soumises au doute : combien d'enfants y a-t-il ? Georges existe-t-il ? Qui est ce narrateur ? Est-il réellement présent ? La fin, qui permet de relire le récit comme un récit de rêve, suggère une lecture symbolique de ces identités flottantes : c'est par l'affirmation de soi face au groupe que les personnages de ce récit s'affirment. Si le silence semble l'affirmation de soi la plus efficace, puisque tout le début du récit tourne autour de Georges, c'est finalement par la parole que les personnages évitent le risque de disparaître.

\section{BIBLIOGRAPHIE}

Blanchot M. 1992. Une voix venue d'ailleurs. Paris. Virgile.

Comina M. 1998. Louis-René Des Forêts, l’impossible silence. Seysel. Champ Vallon.

Des Forêts L.-R. 1960. La Chambre des enfants. Paris. Gallimard.

Rabaté D. 1991. Louis-René des Forêts : La Voix et le Volume. Paris. Corti.

Roudault J. 1995. Louis-René des Forêts. Paris. Seuil.

Quignard P. 1985. Le vœu de silence, essai sur Louis René des Forêts. Montpellier. Fata Morgana.

Première parution dans la revue Granit en 1980.

\section{"Heavy and white silence as a growing anger": to embodie the silence in The Children's Chamber by Louis-René des Forêts}

ABSTRACT: This article try to demonstrate how the silence takes shape: it becomes a dangerous character against whom the narrator has to act. In The Children's Chamber, the silence is at first an ally, helping the young Georges to resist to the other children who want him to talk. Then it becomes a dangerous character who takes more and more space: the adulte who spies the children from the begining of the novel has to interrumpt their game in order to stop the silence.

Keywords: power of silence, loneliness, mutism, childhood. 